\title{
Cardiac Motion Analysis from Ultrasound Sequences Using Non-rigid Registration
}

\author{
María J. Ledesma-Carbayo ${ }^{1}$, Jan Kybic ${ }^{2}$, Manuel Desco ${ }^{3}$, Andrés Santos ${ }^{1}$, and \\ Michael Unser ${ }^{2}$ \\ 1 ETSI Telecomunicación, \\ Universidad Politécnica de Madrid, \\ Ciudad Universitaria s/n 28040 Madrid, Spain \\ mledesma@die.upm.es \\ 2 Department of Microengineering, \\ Swiss Federal Institute of Technology Lausanne, \\ CH-1015 Ecublens, Switzerland \\ Jan.Kybic@epfl.ch \\ 3 Unidad de Medicina y Cirugía Experimental, \\ Hospital General Universitario Gregorio Marañón, \\ Dr. Esquerdo 46, E-28007 Madrid, Spain \\ desco@mce.hggm.es
}

\begin{abstract}
In this article we propose a cardiac motion estimation technique that uses non-rigid registration to compute the dense cardiac displacement field from 2D ultrasound sequences. Our method employs a semi-local deformation model which provides controlled smoothness. We apply a multiresolution optimization strategy for better speed and robustness. To further improve the accuracy, the sequence is registered in both forward and backward directions. We calculate additional parameters from the displacement field, such as total displacement and strain.

We create an artificial ultrasound sequence of one heart cycle using a motion model and use it to validate the accuracy of the algorithm. Finally, we present results on real data from normal and pathological subjects that show the clinical applicability of our method.
\end{abstract}

\section{Introduction}

Cardiac motion estimation constitutes an important aid for the quantification of the elastic and contractility properties of the myocardium. Localized regions with movement abnormalities are related to the existence of ischemic segments, damaged by insufficient tissue microcirculation.

MR imaging and especially tagged MR are currently the reference modalities to estimate dense cardiac displacement fields with high spatial resolution. The deformation fields, as well as derived parameters such as myocardial strain, can be found with good accuracy [1, 2, 3].

Nuclear modalities, such as SPECT and PET were used [4] despite their low spatial and temporal resolutions, high cost and the requirement for specialized 
equipment [5, 6]. Computed tomography has also been considered [7, 8] but has the drawback of high patient radiation dose.

Echocardiography is currently the imaging method most widely used to assess cardiac function. It offers significant advantages over the rest of the imaging techniques: availability, portability, low cost, and no adverse secondary effects. While new ultrasound techniques provide high image quality, increasing attention is being paid to the automatic processing of ultrasound image sequences $[8,9,10,11$.

Many of the aforementioned approaches to cardiac motion analysis start by the segmentation of the myocardial wall, followed by geometrical and mechanical modelling using active contours or surfaces to extract the displacement field and to perform the motion analysis $[8,9,3$. Some authors introduce temporal modelling of the cardiac motion to provide temporal smoothness and better motion tracking [1, 2]. Alternative methods use energy-based warping and optical flow techniques to compute the displacement of the myocardium [5, 6, 7].

For echocardiography, deformable and mechanical model-based techniques are the most popular. They require a presegmentation step which is particularly difficult in the case of cardiac ultrasound images due to the noise and the complexity of cardiac structure [8, 9, 10]. Speckle tracking techniques have also been proposed to estimate heart motion [11.

Here we propose to compute the dense cardiac displacement field from ultrasound sequences using a non-rigid registration algorithm based on a global pixel-based matching criterion. To the best of our knowledge, this kind of approach has not been pursued before, except for the simpler case of M-mode data where dynamic programming-based unidimensional registration has been used [12. We present a fully automatic method that does not require segmentation. We use a semi-local deformation model that provides controlled smoothness of the motion field. Multiresolution strategy yields speed and robustness with respect to the speckle changes in time. While we deploy the methodology for bidimensional ultrasound sequences, it is also applicable to $3 \mathrm{D}$ data.

The accuracy of the algorithm was validated using an artificial heart motion model. Real data from normal and pathological subjects were also analysed to show the clinical applicability of the method.

\section{Methodology}

\subsection{Non-rigid Registration Method}

The workhorse of our approach is an elastic registration algorithm. Given a reference image $f_{r}$ and a test image $f_{t}$, it finds a correspondence function $\mathbf{g}$, which relates coordinates in $f_{t}$ and $f_{r}$. More specifically, we consider registration as a minimization problem. We search a correspondence function $\mathbf{g}: \mathbb{R}^{2} \rightarrow \mathbb{R}^{2}$, such that the warped test image $f_{w}(\mathbf{x})=f_{t}(\mathbf{g}(\mathbf{x}))$ is as close as possible to the reference image $f_{r}$. We measure the quality of the fit using a sum of squared differences (SSD) criterion 


$$
E=\sum_{\mathbf{i} \in I} e_{\mathbf{i}}^{2}=\sum_{\mathbf{i} \in I}\left(f_{w}(\mathbf{i})-f_{r}(\mathbf{i})\right)^{2}
$$

where the summation is taken over all the pixels in the reference image. We generate a continuous version $f_{t}^{c}$ of the discrete image $f_{t}$ by spline interpolation.

$$
f_{t}^{c}(\mathbf{x})=\sum_{\mathbf{i} \in I} b_{\mathbf{i}} \beta_{q}(\mathbf{x}-\mathbf{i})
$$

where $\beta_{q}(\mathbf{x})$ is a tensor product of centered B-splines of degree $q$. It has the advantage of good accuracy and the possibility of evaluating spatial derivatives analytically. We represent the correspondence function $\mathbf{g}$ using splines as well.

$$
\mathbf{g}(\mathbf{x})=\mathbf{x}+\sum_{\mathbf{j} \in \mathbb{Z}^{N}} \mathbf{c}_{\mathbf{j}} \beta_{r}(\mathbf{x} / h-\mathbf{j})
$$

Therefore $\mathbf{g}$ is a linear combination of basis functions $\beta_{r}(\mathbf{x})$ placed on a rectangular grid. The scale parameter $h$ governs the node spacing, the total number of parameters $\mathbf{c}_{\mathbf{j}}$, and the smoothness of the solution. The advantages of this model are good approximation properties, fast evaluation of the deformation, local influence of the parameters, and automatically imposed smoothness.

The resulting problem of optimising (II) with respect to the coefficients $\mathbf{c}_{\mathbf{j}}$ is solved using a standard multidimensional optimisation algorithm. We found that a Marquardt-Levenberg-like algorithm was fastest with respect to the number of iterations, while simple gradient descent-like optimizer converged in the least amount of time.

The original idea of the algorithm was described in [13 and its extensions to multiple dimensions in [14. For the present application, the algorithm was completely redesigned, resulting in a major speed-up. It now registers $256 \times 256$ images pixels in less than $10 \mathrm{~s}$ with subpixel precision on a standard PC. The time required is essentially proportional to the number of pixels.

For the echocardiographic sequences we typically use a control grid of $8 \times 8$ points, represent the deformation using quadratic or cubic splines, and the image using cubic or linear splines.

\subsection{Extraction of the Displacement Field}

The input to our algorithm is an ultrasound sequence of a cardiac cycle composed of $N$ images (frames). We apply the registration algorithm to consecutive pairs of frames within the sequence. The correspondence function $\mathbf{g}$ from (3) provides the displacement field between images $i$ and $i+1$ which we denote $\mathbf{r}_{i, i+1}^{f}$. That is, a point at position $\mathbf{x}$ in image $i$ moves to position $\mathbf{x}+\mathbf{r}_{i, i+1}^{f}(\mathbf{x})$ in image $i+1$. The cumulative displacement field along the sequence for a given frame $i+1$ is then computed as

$$
\mathbf{r}_{0, i+1}^{f}=\mathbf{r}_{0, i}^{f}+\mathbf{r}_{i, i+1}^{f} \quad \text { with } \quad \mathbf{r}_{0,0}^{f}=0
$$


where for brevity we have omitted the spatial coordinates $\mathbf{x}=(x, y)$. As our sequence is cyclic, $\mathbf{r}_{0, N}^{f} \equiv \mathbf{r}_{0,0}^{f}$ should be equal to zero. In practice, we find this error to be small, typically about one pixel. For even better precision, we carry on the registration backward, yielding $\mathbf{r}_{i, i-1}^{b}$ and a cumulative field

$$
\mathbf{r}_{0, i}^{b} \equiv \mathbf{r}_{N, i}^{b}=\mathbf{r}_{N, i+1}^{b}+\mathbf{r}_{i+1, i}^{b}
$$

If we consider the error distribution of the registration as independent, identically distributed and normal, then the maximum likelihood (ML) estimate of the cumulative displacement is

$$
\mathbf{r}_{0, i}=\omega_{i} \mathbf{r}_{0, i}^{f}+\left(1-\omega_{i}\right) \mathbf{r}_{0, i}^{b} \quad \text { with } \quad \omega_{i}=\frac{N-i}{N}
$$

\subsection{Spatio-temporal Derived Parameters}

Once the displacement field is obtained, other parameters of clinical interest are computed. We calculate the velocity and acceleration fields

$$
\mathbf{v}_{i}=\frac{\partial \mathbf{r}_{i}}{\partial t} \quad \mathbf{a}_{i}=\frac{\partial^{2} \mathbf{r}_{i}}{\partial t^{2}}
$$

We compute also the Lagrangian strain, which provides information about myocardial contractility [15] 5]. Strain is defined as the spatial gradient $\varepsilon=\nabla_{\mathbf{x}} \mathbf{r}$ where $\varepsilon$ is the strain tensor, with diagonal terms $\partial r_{x} / \partial x$ and $\partial r_{y} / \partial y$ corresponding to normal directional strains, and antidiagonal terms corresponding to shear strains. The spline representation (3) allows for an analytical computation of the strain.

\subsection{Simulated Sequence Model}

An artificial ultrasound sequence has been generated to validate the algorithm. The sequence is generated by warping an end-diastole apical view image using cubic spline interpolation (2). We used the following motion model

$$
\mathbf{r}_{0, i}(\mathbf{x})=\left[\begin{array}{c}
\sin ^{2}(i \pi / T) a_{x} \sin \frac{\pi\left(x_{0}-x\right)}{2\left|x_{\max }-x_{0}\right|} \\
\sin ^{2}(i \pi / T) a_{y}
\end{array}\right]^{T}
$$

where $i$ is the frame index and $x_{0}$ is the coordinate of the left ventricular long axis, which is oriented vertically; i.e., parallel to axis $y$. We corrupted the deformed images by a multiplicative Rayleigh noise $\eta_{m}$ representing speckle changes, and an additive Gaussian noise $\eta_{a}$ to simulate acoustic attenuation [16, 17 .

$$
n(\mathbf{x})=\eta_{m} \sqrt{f(\mathbf{x})}+\eta_{a} \quad \text { where } \quad \eta_{a} \sim \mathcal{N}(0, \sigma), \eta_{m} \sim \mathcal{R}(\alpha)=\frac{z}{\alpha^{2}} e^{-z^{2} / 2 \alpha^{2}}
$$

and $f(\mathbf{x})$ is the original image. For our images in the $0 \sim 255$ range, we used $\sigma=20$ and $\alpha \approx 0.8$, which corresponds to $\mathrm{E}\left[\eta_{m}\right]=1$. 


\subsection{Real Sequences}

Real data from normal volunteers and ischemic patients were acquired with a Siemens-ACUSON Sequoia ${ }^{\circledR}$ scanner (Mountain View, CA, USA). Two and four chamber view sequences of the left ventricle were analysed.

\section{$3 \quad$ Experiments and Results}

\subsection{Simulated Sequence}

This section presents the results with the artificial sequence described in Section 2.4. First, a series of experiments was conducted to choose the most suitable parameters for the registration algorithm. Figure 1 shows the accumulated displacement and velocity fields as small arrows superimposed on the ultrasound images.
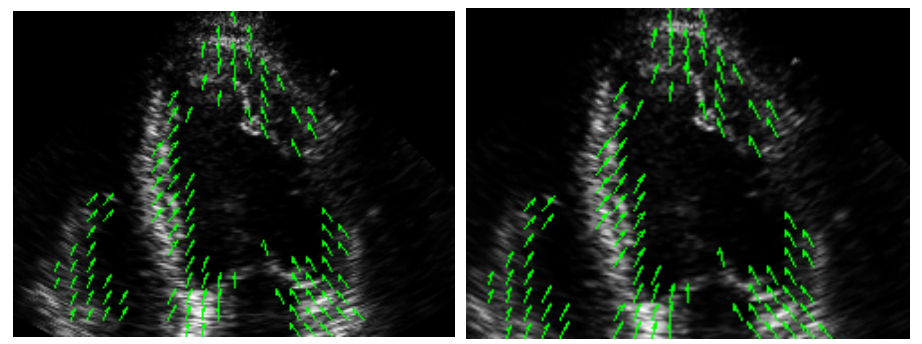

Fig. 1. Simulated sequence results. Left: Accumulated displacement field at the time of maximum contraction $(t=320 \mathrm{~ms})$. Right: Velocity field during contraction $(t=120 \mathrm{~ms})$.

Figure 2 shows the axial and longitudinal accumulated displacements for a middle septum point (a point in the middle of the vertical wall on the left in the images). Even in this noisy case we found good agreement between the true movement and the movement found by the algorithm. The mean square error over the whole sequence for 25 selected points within the myocardium was $1.3 \mathrm{~mm}$.

\subsection{Real Sequences}

In this section, we describe experiments on real sequences from normal volunteers and ischemic patients. Figure 3 shows the computed displacement field vectors of two chamber view sequences from a normal volunteer and an ischemic patient. The ischemic patient presents an infarct in the inferior wall with severe hypokinesis and a normokinetic basal aneterior segment. For the healthy volunteer the accumulated displacement observed corresponds to normal left ventricular contraction. On the other hand the analysis of the ischemic patient 

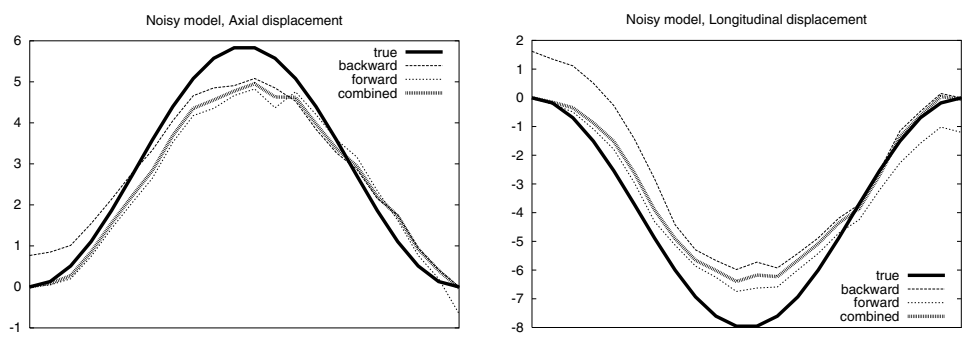

Fig. 2. Simulated sequence results. Axial (left) and longitudinal (right) displacements in mm for a middle septum point. Real displacements, results of the forward and backward registration, and the combined result.

confirms the pathological function of the inferior wall, having very small movement, and quasi normal motion of the basal anterior segment. These results are also shown quantitatively in Figure 4 that represents the axial and longitudinal displacement of a point in the medial inferior segment for the normal and the ischemic cases.
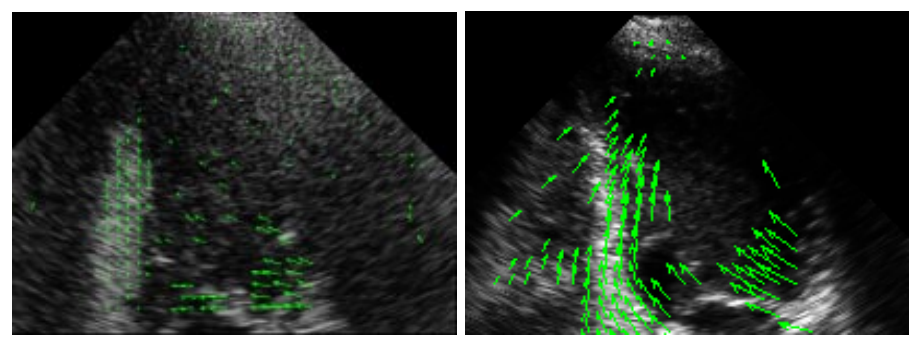

Fig. 3. Accumulated displacement field at the mechanical systole for a normal subject (right) and an ischemic patient with inferior infarct (left).

Figure 5 shows the results of the analysis of a four chamber sequence from an ischemic patient with hypokinetic function of the lateral wall and basal and distal septum segments. The displacement field correlates well with the diagnosis. Strain analysis shows constantly low lagrangian longitudinal strain and normal axial strain for the medial and distal septum segments.

\section{Conclusions}

We have presented a fully automatic method to compute myocardial displacement and deformations from ultrasound sequences. The method has been validated on simulated and real sequences. Results show that the proposed method is able to estimate heart motion and to provide plausible displacement and velocity fields. The results of applying the method to data from normal and ischemic 

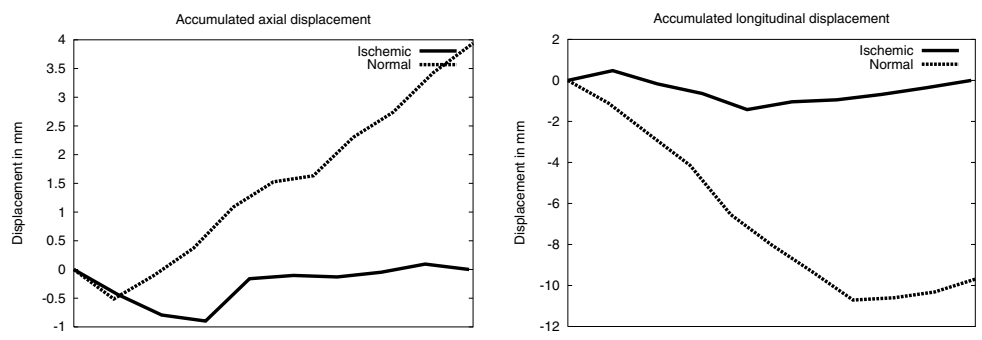

Fig. 4. Axial and longitudinal systolic displacements of a medial inferior segment point for a normal volunteer and an ischemic patient with inferior infart.
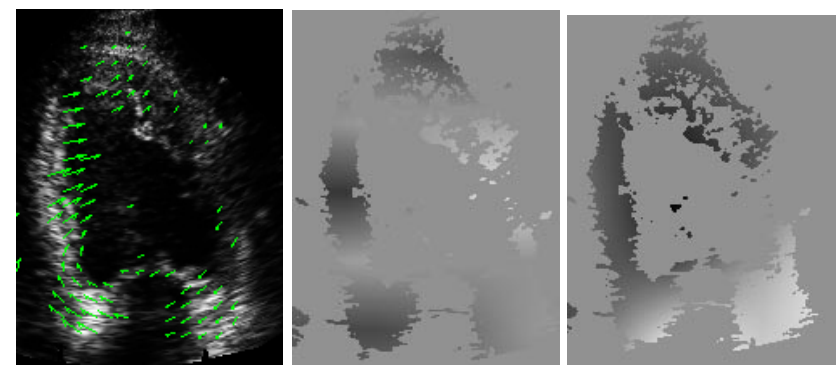

Fig. 5. Accumulated displacement for a four chamber sequence of an ischemic patient (left). Axial (middle) and longitudinal (right) strain images for midsystole $(t=120 \mathrm{~ms})$.

patients is promising and encourages clinical applicability. Further clinical validation is planned for the immediate future.

\section{Acknowledgements}

This work was supported in part by grant No 3200-059517.99/1 from the Swiss Science Foundation, and the Spanish projects TIC99-1085 and III-PRICYT.

\section{References}

[1] P. Clarysse, C. Basset, L. Khouas, P. Croisille, D. Friboulet, C. Odet, and I. Magnin, "Two-dimensional spatial and temporal displacement and deformation field fitting from cardiac magnetic resonance tagging," Medical Image Analysis, vol. 4, no. 4, pp. 253-268, 2000.

[2] J. C. McEachen, A. Nehorai, and J. S. Duncan, "Multiframe temporal estimation of cardiac nonrigid motion," IEEE Trans. Med. Imag., vol. 9, pp. 651-664, April 2000.

[3] P. Shi, A. J. Sinusas, R. T. Constable, and J. S. Duncan, "Volumetric deformation analysis using mechanics-based data fusion: applications in cardiac motion 
recovery.," International Journal of Computer Vision, vol. 35, no. 1, pp. 87-107, 1999.

[4] P. Brigger, S. Bacharach, G. Srinivasan, K. Nour, J. A. Carson, V. Dilsizian, A. Aldroubi, and M. Unser, "Segmentation of gated T1-SPECT images and computation of ejection fraction: A different approach," Journal of Nuclear Cardiology, vol. 6, pp. 286-297, May-June 1999.

[5] J. Declerck, J. Feldmar, and N. Ayache, "Defininion of a 4D continuous planispheric transformation for the tracking and the analysis of left-ventricle motion," Medical Image Analysis, vol. 2, no. 2, pp. 197-213, 1998.

[6] J.-P. Thirion and S. Benayoun, "Myotrack: A 3D deformation field method to measure cardiac motion from gated SPECT," in Proc. MICCAI 2000, Lecture Notes in Computer Science, vol. 1935 (B. J. Scott L. Delp, Anthony M.DiGioia, ed.), pp. 697-706, Springer Verlag, Berlin, Oct. 2000.

[7] J.-M. Gorce, D. Fibroulet, and I. Magnin, "Estimation of three dimensional cardiac velocity fields: assessment of a diferential method and application to threedimensional CT data," Medical Image Analysis, vol. 1, no. 3, pp. 245-261, 1996/7.

[8] C. Nastar and N. Ayache, "Frequency-based nonrigid motion analysis application to four dimensional medical images," IEEE Trans. Pattern Anal. Mach. Intell., vol. 18, pp. 1067-1079, November 1996.

[9] G. Jacob, A. Noble, M. Mulet-Parada, and A. Blake, "Evaluating a robust contour tracker on echocardiographic sequences," Medical Image Analysis, vol. 3, no. 1, pp. 63-75, 1999.

[10] X. Papademetris, A. J. Sinusas, D. P. Donald, and J. S. Duncan, "Estimation of 3D left ventricular deformation from echocardiography," Medical Image Analysis, vol. 5, pp. 17-28, March 2001.

[11] F. Yeung, F. Levinson, D. Fu, and K. J. Parker, "Feature-adaptive motion tracking of ultrasound image sequences using a deformable mesh," IEEE Trans. Med. Imag., vol. 17, pp. 945-956, Dec. 1998.

[12] M. Unser, G. Pelle, P. Brun, and M. Eden, "Computer analysis of M-mode echocardiograms: estimation of spatial deformation with time," in Cardiovascular Dynamics and Models, pp. 304-310, Paris: Institut National de la Santé et de la Recherche Médicale, 1988.

[13] J. Kybic, P. Thévenaz, A. Nirkko, and M. Unser, "Unwarping of unidirectionally distorted EPI images," IEEE Trans. Med. Imag., vol. 19, pp. 80-93, Feb. 2000.

[14] J. Kybic and M. Unser, "Multidimensional elastic registration of images using splines," in Proceedings of ICIP, (Vancouver, Canada), 2000.

[15] J. D'hooge, A. Heimdal, F. Jamal, T. Kukuslki, R. F. Bijnens, B. and, L. Hatle, P. Suetens, and G. Sutherland, "Regional strain and strain rate measurements by cardiac ultrasound: Principles, implemantation and limitations.," Eur J. Echocardiography, vol. 1, pp. 154-170, 2000.

[16] X. Zong, A. Laine, and E. Geiser, "Speckle reduction and contrast enhancement of echocardiograms via multiscale nonlinear processing," IEEE Trans. Med. Imag., vol. 17 , no. 4, pp. 532-540, 1998.

[17] T. Loupas, W. McDicken, and P. Allan, "An adaptive weighted median filter for speckle suppression in medical ultrasonic images," IEEE Trans. Circ. Syst., vol. 36, pp. 129-135, Jan. 1989. 